Running Head: HOUSEHOLD STRUCTURE AND MENTAL DISTRESS DURING COVID-19

\title{
Associations of Household Structure and Presence of Children in the Household with Mental Distress during the COVID-19 Pandemic
}

Emily Smail, BSc ${ }^{1}$, Kira Riehm, $\mathrm{MSc}^{1}$, Cindy Veldhuis, $\mathrm{PhD}^{2}$, Renee M. Johnson, $\mathrm{PhD}^{1}$, Calliope Holingue, $\mathrm{PhD}^{1,3}$, Elizabeth Stuart, $\mathrm{PhD}^{1}$, Luke Kalb, $\mathrm{PhD}^{1,3}$, Johannes Thrul, $\mathrm{PhD}^{1,4}$

1. Department of Mental Health, Bloomberg School of Public Health, Johns Hopkins University; 624 N Broadway, Baltimore, MD 21205, USA

2. School of Nursing, Columbia University; $560 \mathrm{~W}$ 168th Street, New York, NY 10032, USA

3. Department of Neuropsychology, Kennedy Krieger Institute, Johns Hopkins University; 707 N Broadway, Baltimore, MD 21205, USA

4. Centre for Alcohol Policy Research, La Trobe University, Plenty Rd \&, Kingsbury Dr, Bundoora VIC 3086, Australia

Corresponding author: Emily Smail; 624 N Broadway, Room 897, Baltimore, MD 21205, esmail1@jhu.edu 


\begin{abstract}
Purpose: The objectives of the current study were to: (1) assess the impact of household structure (i.e. living alone compared to living with children, a partner, or both) and presence of children on mental distress during the COVID-19 pandemic; and (2) determine whether these associations are moderated by income or sex.
\end{abstract}

Results: A total of 2,524 adults aged 25-55 were included in the analytic sample. Multivariable linear regression models were used to examine associations between explanatory variables and mental distress, measured via the Patient Health Questionnaire (PHQ)-4. In adjusted models, each additional child under the age of 12 was associated a decrease in mental distress $(\beta=-0.21, p=0.03)$. This finding was significant when adjusting for sociodemographic variables (age, sex, race, education, household income, living with a partner, and currently having a job) and historical depressive symptoms (CESD-8). Having children between the ages of 13 and 18 and household structure were not significantly associated with mental distress.

Conclusion: This study supports prior literature that demonstrates the positive association of childrearing with psychological well-being, and suggests that these benefits may be present even under stay-at-home orders in the COVID-19 pandemic.

Keywords: mental distress; COVID-19; family structure; parenting paradox 


\section{Introduction}

The emergence of COVID-19 in the United States has created unprecedented challenges that have rendered many individuals financially unstable (Goodell, 2020) and emotionally vulnerable (Li et al., 2020). The well-being of families may be particularly at risk as a result of changes in relationships and routines during COVID-19, which may disproportionately affect children's coping abilities and parental burden (Prime, Wade, \& Browne, 2020; Russell, Hutchison, Tambling, Tomkunas, \& Horton, 2020). Although emerging evidence suggests that there has been a rise in mental distress throughout the pandemic (Kirzinger, Kearney, Hamel, \& Brodie, 2020), there is not, to our knowledge, any existing research on the association between household structure and mental distress. Understanding these associaitons is key to informing public health planning efforts.

Recent polls have highlighted the distressing nature of the pandemic (Holingue, Kalb, \& Riehm, In Press). Data from the Kaiser Family Foundation poll found that nearly half of adults in the US are experiencing negative mental health effects from worry and stress over the virus (Kirzinger et al., 2020; McGinty, Presskreischer, Han, \& Barry, 2020). The COVID-19 pandemic has increased the amount of time that many adults are spending at home, which could have varying implications for mental health depending on household structure, such as living with children, partners, both, or neither. People living alone may be particularly at risk for

experiencing loneliness during the COVID-19 pandemic (McGinty et al., 2020). Among young (ages 16-34) and middle-aged (ages 35-59) adults, living alone has been associated with an increased risk for common mental disorders (Jacob, Haro, \& Koyanagi, 2019), including a nearly two-fold increase in anxiety and depressive disorders, compared to those who are married or cohabiting (Joutsenniemi, Martelin, Martikainen, Pirkola, \& Koskinen, 2006). Families with 
children may also be disproportionately affected as most daycares and almost every school across the US has closed. Classes have shifted to home-based, distance-learning models, affecting nearly 55 million students in kindergarten through $12^{\text {th }}$ grade (Golberstein, Wen, \& Miller, 2020). In addition to everyday parenting, many parents quickly assumed the role of primary educator while potentially balancing competing demands from employment. The experience of COVID-19 related stressors, coupled with symptoms of anxiety and depression, has been linked to higher parental perceived stress (Brown, Doom, Lechuga-Pena, Watamura, \& Koppels, In Press.; Spinelli, Lionetti, Pastore, \& Fasolo, 2020).

Notably, however, having children in the home can be associated with both positive and negative mental outcomes. This phenomenon is known as the parenting paradox ; though (Rizzo, Schiffrin, \& Liss, 2013) childcare is demanding and time-consuming, parents tend to value their time with children as it promotes familial connectedness and has high intrinsic value for the parent (Bianchi, Robinson, \& Milkie, 2006). The latter is particularly true of interactive childcare, such as playing with a child, as opposed to routine care such as feeding or bathing a child (Offer, 2014). Previous research highlights that the type of interaction may be highly gendered (McDonnell, Luke, \& Short, 2019) and may have discrete implications for wellbeing by the sex of the parent. For example, routine childcare is more stressful and less engaging for mothers than it is for fathers (Offer, 2014) and these associations may further depend on the age of the child (Roeters \& Gracia, 2016). Stress levels for other forms of childcare are comparable among parents of both genders. Thus, studies investigating associations between caregiving for children during the COVID-19 pandemic and mental distress should account for sex of the parent. 
The stresses of childrearing are likely amplified among single parents, partially due to lower levels of financial and emotional support (Sobolewski \& King, 2005). These lower levels of support may result in poorer mental health for single parents compared to parents who are married or cohabiting (Crosier, Butterworth, \& Rodgers, 2007). These challenges may be particularly relevant during the COVID-19 pandemic, as single parents may be unable to rely on supportive family and friends throughout shelter-in-place orders.

Finally, income inequalities have repeatedly been linked to psychological outcomes (Patel et al., 2018; Pickett, James, \& Wilkinson, 2006). These inequalities are likely to be widened during the COVID-19 pandemic as a result of job loss (U.S. Department of Labor, 2020). Indeed, data from a recent poll suggest that individuals belonging to a low-income household (less than $\$ 40,000$ per year) have disproportionately experienced negative impacts to their mental health during the COVID-19 pandemic compared to those who make $\$ 40,000$ or more (Kirzinger et al., 2020).

As the world enters a new phase with COVID-19, many novel stressors are emerging. Families are faced with particular challenges of additional child rearing and educational duties and people who live alone may experience distress due to isolation. The added responsibilities of childcare may be particularly distressing for women, who have historically provided the bulk of care. Further, households with low economic resources may experience high levels of stress, and the financial effects may compound stress differently based on household structure. To our knowledge, however, no research has examined mental distress and household structure during the pandemic. To address this gap, the objectives of this study are to: (1) assess the impact of household structure (i.e. living alone compared to living with children, a partner, or both) and 
presence of children on mental distress during the COVID-19 pandemic; and (2) determine whether these associations are moderated by income or sex.

\section{Methods}

Study Sample

Data for this study come from the Understanding America Study (UAS). UAS participants were selected using Address Based Sampling (ABS), in which postal records are used to select a random sample from a listing of residential addresses (Lavrakas, 2008). Eligible individuals include adults 18 and older in the contacted households. The UAS panel consists of 10 nationally representative cohorts (the University of Southern California, 2020) enrolled in the sample between 2014 and 2020. The current analysis uses data from the UAS 235 wave (April 2020 Monthly Survey, administered April $1^{\text {st }}$ through April 28 ${ }^{\text {th }}$ ), which assesses participant responses to the COVID-19 pandemic. Survey weights in the UAS align sample distributions of key demographic characteristics (e.g., sex, race/ethnicity) to their population counterparts based on the Basic Monthly Current Population Survey (CPS).

\section{Measures}

\section{Mental Distress}

The primary outcome measure was the 4-item version of the Patient Health Questionnaire (PHQ-4). This measure is a brief self-report questionnaire consisting of 2 items that assess depressive symptoms and 2 items that assess anxiety symptoms over a two-week period (Löwe et al., 2010). Response options include "not at all," "several days," "more than half the days," and "nearly every day." The total score is calculated by adding the scores of all 4 items; higher scores represent greater levels of mental distress (Kroenke, Spitzer, Williams, \& Lowe, 2009). 


\section{Historical Depressive Symptoms}

In earlier waves of data collections, participants completed the 8-item version of the Center for Epidemiologic Studies-Depression Scale (CESD-8) (Bracke, Levecque, \& Van de Velde, 2008; Radloff, 1977). Answers range from 0 (none or almost none of the time) to 3 (all or almost all the time). The number of symptoms (count: $0-8$ ) a respondent previously endorsed as occurring "all or almost all the time" in the past 7 days was used as a historical measure of depressive symptoms. The most recent CESD-8 was used for participants with multiple CESD-8 measurements from prior waves (46\% of the sample had CESD-8 from June 2019, 36\% from June 2017, and 18\% from May 2015). Continuously measured CESD-8 scores were included as a covariate in all models.

\section{Household Variables}

The primary exposure of interest was household structure, which was assessed using the respondent-reported relationships of household members. Household structure was assigned to the following categories: living alone; living with spouse only; living with children only; living with spouse and children only; living with significant other only; living with significant other and children only. All other combinations of household structure (22.7\% of households), including extended family and non-family members, were not assessed due to the heterogeneity of this population. In addition, household structure was further characterized by identifying the number of children living in the household. Children were split into two age groups, children aged 12 or below (range: $0-6$ ) and children aged 13 to 18 (range: $0-4$ ).

\section{Other Sociodemographic Items}

Sociodemographic factors included: age category (in years); sex (female or male); race/ethnicity [White, Black or African American, or Other (includes American Indian or Alaska 
Native, Asian, Native Hawaiian or Pacific Islander, and multiracial)]; education (high school degree or below, attended some college or received a two-year degree, Bachelor's degree, or graduate degree); household income, measured as the total combined 12-month income of all members currently living the respondent's household (less than 30k, 30-59k, 60k and above); and currently have a job (yes or no). A household income of $\$ 60,000$ was selected as the cutoff for low versus high income as it roughly corresponds to the median income in the U.S. in 2018 (Guzman, 2019).

\section{Statistical Analyses}

Multivariable, survey-weighted linear regression was used to examine the associations of household characteristics (i.e., household structure and number of children) as explanatory variables and total PHQ-4 score as the outcome. The analysis was restricted to individuals with complete information on all analytic variables between the ages of 25 and 55 (48 participants $[\sim 2 \%]$ excluded for incomplete data). Age restrictions were implemented to reduce confounding by age; age categories (by decade, beginning at age 25) were selected if greater than one-third of the group had children in the home. We then estimated two additional models with an interaction term of each primary predictor (household structure and number of children) with sex and household income separately.

Sensitivity analyses were included to allow for potentially different results based on distinct ways of measuring a key construct (i.e., household income versus COVID-related financial instability), threshold (i.e., cutoff for low income), similarities of living situation (i.e., living with spouse and living with significant other) and comparison group (i.e., living alone versus living with spouse only as reference group in the household structure model and adjusting for caregiving among individuals with children in the model assessing number of children). All 
analyses used the UAS survey weights and adjusted for within-household clustering, allowing these results to generalize to the US adult population. All analyses were performed in Stata 15 using the svy commands (Statacorp, 2017).

\section{Results}

\section{Sample Description}

A total of 2,524 adults, ages 25-55, were included in this analysis. The majority of the participants were female (53\%), white (76\%), currently working (66\%), and living with a partner (73\%). Most had some post-secondary education (65\%) and an annual income of greater than $\$ 60,000$ (52\%). CESD-8 total scores ranged from 0 to 8 with a mean of $1.9(\mathrm{SE}=0.062)$. Over one-third of participants endorsed no CESD-8 symptoms at their most recent measurement, and only $20 \%$ reported experiencing more than 3 of the symptoms. Sample characteristics are presented in Table 1. PHQ-4 total scores ranged from 0 to 12 with a mean of 2.9 ( $\mathrm{SE}=0.091)$. Though the majority of participants (55\%) fell within normal levels of distress, many participants reported mild (26\%), moderate (11\%), and severe ( $8 \%$ ) levels of distress.

\section{Correlates of Mental Distress}

\section{Unadjusted Analyses}

In unadjusted bivariate tests, neither of the primary predictor variables (i.e., household structure and number of children, stratified by age) were associated with mental distress (i.e., PHQ-4 total scores). Prior CESD-8 scores were associated with higher mental distress $(\mathrm{p}<0.05)$. Higher income and currently having a job had a negative association with mental distress $(\mathrm{p}<0.001)$. Older age (45-55 compared to 25-34), being male, and being Black/African American (compared to White) were associated with significantly lower lower mental distress (all $\mathrm{p}<0.005$ ) in the unadjusted models. 


\section{Adjusted and Stratified Analyses}

In adjusted models, compared to households with no children, each additional child under the age of 12 was associated a decrease in mental distress $(\beta=-0.21, p=0.03)$ (Table 2, Figure 1). This finding was significant when adjusting for sociodemographic variables (age, sex, race, education, household income, living with a partner, and currently having a job) and historical CESD-8 score. Having children between the ages of 13 and 18, number of household members, and household structure were not significantly associated with mental distress. The strength of association and significance of other variables varied across models (household structure and number of children), but generally, being female, currently not working, and historical depressive symptoms were associated with higher levels of mental distress. Black/African American, compared to White individuals, had significantly lower mental distress in all models $(\beta=-1.06, p<0.001)$. A summary of these results is available in Table 2. Additional models included interaction terms between the primary exposures (i.e., household structure and number of children) and sex and income to investigate differential effects among men/women and high/low income groups. These interaction effects were not statistically significant. A summary of these results is available in Tables 3 and 4.

\section{Sensitivity Analyses}

Results did not change when using a COVID-related indicator of financial instability ("In the past seven days, were you worried you would run out of food because of a lack of money or other resources?"), though responding “yes" to this question significantly influences PHQ-4 score $(B=2.25, p<0.001$ in unadjusted models). Additionally, using other household income cutoffs (i.e., \$30k, \$50k) and other reference categories for household structure (e.g., living with spouse versus living alone) did not change the results. Similarly, combining categories of 
comparable living situations, namely, living with spouse and living with significant other and living with spouse or significant other and children, did not change the association with total PHQ-4 score.

\section{Discussion}

Our findings, based on data collected during the April 2020 wave of a nationally representative survey panel, found no significant differences in mental distress in individuals living alone compared to individuals living with family members or significant others (e.g., spouse or spouse and children). This finding is consistent with early literature that proposes no differences in mental health symptoms based on household structure (Cramer, 1993), but is in contrast to recent studies that suggest that individuals living alone fare worse than those living with others (Jacob et al., 2019). It may be that individuals living alone have found unique ways to stay connected during this time (e.g., virtual hangouts, socially distant walks in the park, etc.) that provide buffers against worsened mental health. Alternatively, people living alone may be less concerned about exposing friends and family to the virus, which has been identified as a significant source of anxiety, particularly among healthcare workers (Shanafelt, Ripp, \& Trockel, 2020). Relatedly, people living alone may be able to practice social distancing more conscientiously than those living with others, which could serve as a source of relief.

This study suggests that having children ages 12 and under in the household is associated with lower mental distress, whereas having children ages 13 to 18 in the household is not associated with mental distress. Findings for this study are consistent with previous literature which suggest that parents experience high levels of well-being when spending time caring for young children (Roeters \& Gracia, 2016). This may be true particularly during the stay-at-home orders, which were enacted almost ubiquitously across all 50 states in April. Many parents were 
able to spend additional time with their children, and, as a result of the caregiving responsibilities, were prompted to implement practices that are known to be beneficial to mental health and resilience, including routine breaks from work, establishing a schedule, improving and increasing communication, and engaging in activities as a family (Prime et al., 2020). For families with dual parents, childcare and household tasks may be more collaborative than before the pandemic, which may also improve wellbeing. Having a family is associated with an increased sense of purpose and responsibility for parents which has positive impacts on mental and physical health (Pollitt, Robinson, \& Umberson, 2018). These effects may be enhanced during the pandemic as a result of increased time spent with children and the need to provide support and a sense of normalcy for children during a highly stressful time.

It is important to note, however, that these effects may not be universally experienced by parents. Recent studies have shown that the impact of COVID-19 among parents varies with caregiver burden, perceptions of children's stress (Russell et al., 2020), and the availability of resources to meet caregiver needs (Griffith, 2020). Therefore, while this study shows that young children, on average, are associated with reduced mental distress among parents, there are likely nuances in the experience of mental distress among parents as a result of these factors and other characteristics of family and work structure (e.g., age of children, fleixibility of job, support system).

Not surprisingly, parents of adolescents are less likely to engage in caregiving activities as children in this age range are increasingly autonomous. While some earlier studies have suggested that rising parent-child tension in adolescence cause parental distress (Laursen, Coy, \& Collins, 1998), there is little empirical evidence showing child-induced distress. Prior studies have suggested numerous possibilities for this lack of evidence, including inter-person variability 
in the experience of demands and rewards of childrearing and the notion that rewards and burdens of childrearing offset each other (Umberson, Pudrovska, \& Reczek, 2010). Further, recent evidence suggests that parents and non-parents have experienced similar levels of disruption to their daily lives during the COVID-19 pandemic (Kirzinger et al., 2020); however, the survey did not take into account age differences of children. It may be that disruption of daily life for parents with children aged 13 to 18 are more comparable to individuals without children in the home, in terms of added childcare and schooling activities, than parents with children at or below the age of 12 .

These data showed no significant interaction of the primary predictor variables (e.g., household structure and number of children) with past-year household income (less than/greater than $\$ 60,000$ ) or sex (female/male), suggesting that the association between household structure and mental distress does not vary by sex or income. In addition, the data showed no main effects of income. There are many plausible explanations for these findings. For example, self-report of household income is prone to measurement error (Moore \& Welniak, 2000) and may not accurately represent one's actual household income, particularly during the COVID-19 pandemic. Further, there is substantial variation in household size (between 0 and 11 housemates reported in this population of UAS participants). As expected, a livable income for one household may not be equivalent to the livable income of another. The COVID-related financial distress variable is thought to capture some of these nuances, asking whether a participant is worried about running out of food. Given the lack of association between mental distress and this financial variable in adjusted models, it may be possible that individuals are encountering fewer expenses during the pandemic (e.g., eating out less, travelling less) (Nicola et al., 2020), which may counter some of the concern brought about by financial insecurity. 
Regarding sex differences, the data show that men, on average, have lower distress scores than females. This reflects general trends of depression and anxiety (Altemus, Sarvaiya, \& Neill Epperson, 2014), and the recent finding that women report disproportionate worry over coronoavirus-related concerns (Frederiksen, Gomez, Salganicoff, \& Ranji, 2020). Anecdotally, many sources hypothesize that men may be taking on additional household responsibilities during the pandemic, including routine caregiving and household chores (Carlson, Petts, \& Pepin, 2020; Levs, 2020; Miller, 2020). More research is needed to determine whether gendered divisions of labor have shifted during the pandemic and if so, whether these have associations with mental health.

Despite many strengths, such as the use of a nationally representative study population, this study is not without limitations. Foremost is the cross-sectional design of the study, which implies that no conclusions can be drawn on the directionality of associations. However, we were able to control for depressive symptoms for a previous wave of the UAS in all analyses. Second, we lacked data on relationship quality, marital satisfaction, and child mental health, which could mediate the association between household variables and mental distress. Investigating these potential mediators is an important direction for future studies.

This study supports previous research suggesting positive effects of childrearing and demonstrates that parents with young children may be particularly likely to experience these benefits (e.g., increased likelihood of taking routine breaks from work, spending time outside, and having set wake and bedtime schedules) (Craig \& Churchill, 2020) during a stay-at-home order. Engaging in these physical and emotional activities, which come naturally with childcare, may also facilitate mental well-being among those not currently providing care. Lack of evidence for differences in mental distress in those living alone versus those living with others 
could signal a uniform response to stress and uncertainty, though further evidence is necessary to confirm this finding. These data are unique in that they capture mental health of the U.S. population approximately one month into the U.S. response to the pandemic at a time when nearly all schools were closed, and all states were under stay-at-home orders. As more data are published, it will be essential to understand the long-term consequences of specific living situations on mental health in order to inform recommendations and practices during these unique times. 


\section{References}

Altemus, M., Sarvaiya, N., \& Neill Epperson, C. (2014). Sex differences in anxiety and depression clinical perspectives. Frontiers in Neuroendocrinology, 35(3), 320-330. doi:10.1016/j.yfrne.2014.05.004.

Bianchi, S., Robinson, J., \& Milkie, M. (2006). Changing rhythms of american family life. New York: Russell Sage Foundation, 6(4).

Bracke, P., Levecque, K., \& Van de Velde, S. (2008). The psychometric properties of the CES-D 8 depression inventory and the estimation of cross-national differences in the true prevalence of depression. Univ Leuven,

Brown, S., Doom, J., Lechuga-Pena, S., Watamura, S., \& Koppels, T. (In Press.). Stress and parenting during the global COVID-19 pandemic. Child Abuse and Neglect., Retrieved from https://doi.org/10.1016/j.chiabu.2020.104699.

Carlson, D. L., Petts, R., \& Pepin, J. (2020). US couples' divisions of housework and childcare during COVID-19 pandemic. SocArXiv, doi:10.31235/osf.io/jy8fn.

Craig, L., \& Churchill, B. (2020). Dual-earner parent couples' work and care during COVID-19. Gender, Work \& Organization, n/a(n/a) doi:10.1111/gwao.12497.

Cramer, D. (1993). Living alone, marital status, gender and health. Journal of Community \& Applied Social Psychology, 3(1), 1-15. doi:10.1002/casp.2450030102. 
Crosier, T., Butterworth, P., \& Rodgers, B. (2007). Mental health problems among single and partnered mothers. the role of financial hardship and social support. Social Psychiatry and Psychiatric Epidemiology, 42(1), 6-13. doi:10.1007/s00127-006-0125-4.

Frederiksen, B., Gomez, I., Salganicoff, A., \& Ranji, U. (2020, -03-20). Coronavirus: A look at gender differences in awareness and actions. Retrieved from https://www.kff.org/coronavirus-covid-19/issue-brief/coronavirus-a-look-at-genderdifferences-in-awareness-and-actions/.

Golberstein, E., Wen, H., \& Miller, B. F. (2020). Coronavirus disease 2019 (COVID-19) and mental health for children and adolescents. JAMA Pediatrics, doi:10.1001/jamapediatrics.2020.1456.

Goodell, J. W. (2020). COVID-19 and finance: Agendas for future research. Finance Research Letters, , 101512. doi:10.1016/j.frl.2020.101512.

Griffith, A. K. (2020). Parental burnout and child maltreatment during the COVID-19 pandemic. Journal of Family Violence, , 1-7. doi:10.1007/s10896-020-00172-2.

Guzman, G. (2019). U.S. median household income up in 2018 from 2017. Retrieved from https://www.census.gov/library/stories/2019/09/us-median-household-income-up-in-2018from-2017.html.

Holingue, C., Kalb, L. G., \& Riehm, K. E. (In Press). Mental distress in the united sates at the beginning of the 2019 coronavirus disease (COVID-19) pandemic. American Journal of Public Health. 
Jacob, L., Haro, J. M., \& Koyanagi, A. (2019). Relationship between living alone and common mental disorders in the 1993, 2000 and 2007 national psychiatric morbidity surveys. PloS One, 14(5), e0215182. doi:10.1371/journal.pone.0215182.

Joutsenniemi, K., Martelin, T., Martikainen, P., Pirkola, S., \& Koskinen, S. (2006). Living arrangements and mental health in finland. Journal of Epidemiology and Community Health, 60(6), 468-475. doi:10.1136/jech.2005.040741.

Kirzinger, A., Kearney, A., Hamel, L., \& Brodie, M. (2020, April). KFF health tracking poll early april 2020: The impact of coronavirus on life in america. Retrieved from https://www.kff.org/coronavirus-covid-19/report/kff-health-tracking-poll-early-april-2020/.

Kroenke, K., Spitzer, R. L., Williams, J. B. W., \& Lowe, B. (2009). An ultra-brief screening scale for anxiety and depression: The PHQ-4. Psychosomatics, 50(6), 613-621. doi:10.1176/appi.psy.50.6.613.

Laursen, B., Coy, K. C., \& Collins, W. A. (1998). Reconsidering changes in parent-child conflict across adolescence: A meta-analysis. Child Development, 69(3), 817-832. doi:10.1111/j.1467-8624.1998.tb06245.x.

Lavrakas, P. J. (2008). Encyclopedia of survey research methods (vols. 1-0). doi:10.4135/9781412963947.

Levs, J. (2020). One upside of COVID-19: Kids are spending more time with dads. Retrieved from https://www.scientificamerican.com/article/one-upside-of-covid-19-kids-are-spendingmore-time-with-dads/. 
Li, Z., Ge, J., Yang, M., Feng, J., Qiao, M., Jiang, R., .. . Yang, C. (2020). Vicarious traumatization in the general public, members, and non-members of medical teams aiding in COVID-19 control. Brain, Behavior, and Immunity, doi:S0889-1591(20)30309-3 [pii].

Löwe, B., Wahl, I., Rose, M., Spitzer, C., Glaesmer, H., Wingenfeld, K., . . B Brähler, E. (2010). A 4-item measure of depression and anxiety: Validation and standardization of the patient health questionnaire-4 (PHQ-4) in the general population. Journal of Affective Disorders, 122(1-2), 86-95. doi:10.1016/j.jad.2009.06.019.

McDonnell, C., Luke, N. K., \& Short, S. E. (2019). Happy moms, happier dads: Gendered caregiving and parents' affect. Journal of Family Issues, 40(17), 2553-2581. doi:10.1177/0192513X19860179.

McGinty, E. E., Presskreischer, R., Han, H., \& Barry, C. L. (2020). Psychological distress and loneliness reported by US adults in 2018 and april 2020. Jama, doi:10.1001/jama.2020.9740.

Miller, C. (2020). Survey finds half of men think they do most homeschooling and 3\% of women agree. Retrieved from https://www.scarymommy.com/women-more-housework-pandemic/.

Moore, J. C., \& Welniak, E. J. (2000). Income measurement error in surveys: A review. Journal of Official Statistics, 16(4), 331. Retrieved from https://search.proquest.com/docview/1266846677.

Nicola, M., Alsafi, Z., Sohrabi, C., Kerwan, A., Al-Jabir, A., Iosifidis, C., . . Agha, R. (2020). The socio-economic implications of the coronavirus pandemic (COVID-19): A review. 
International Journal of Surgery (London, England), 78, 185-193.

doi:10.1016/j.jjsu.2020.04.018.

Offer, S. (2014). Time with children and employed parents' emotional well-being. Social Science Research, 47, 192-203. doi:10.1016/j.ssresearch.2014.05.003.

Patel, V., Burns, J. K., Dhingra, M., Tarver, L., Kohrt, B. A., \& Lund, C. (2018). Income inequality and depression: A systematic review and meta-analysis of the association and a scoping review of mechanisms. World Psychiatry, 17(1), 76-89. doi:10.1002/wps.20492.

Pickett, K. E., James, O. W., \& Wilkinson, R. G. (2006). Income inequality and the prevalence of mental illness: A preliminary international analysis. Journal of Epidemiology and Community Health, 60(7), 646-647. doi:10.1136/jech.2006.046631.

Pollitt, A. M., Robinson, B. A., \& Umberson, D. (2018). Gender conformity, perceptions of shared power, and marital quality in same- and different- sex marriages. Gender \& Society: Official Publication of Sociologists for Women in Society, 32(1), 109-131. doi:10.1177/0891243217742110.

Prime, H., Wade, M., \& Browne, D. T. (2020). Risk and resilience in family well-being during the COVID-19 pandemic. The American Psychologist, doi:10.1037/amp0000660.

Radloff, L. S. (1977). The CES-D scale. Applied Psychological Measurement, 1(3), 385-401. doi:10.1177/014662167700100306. 
Rizzo, K., Schiffrin, H., \& Liss, M. (2013). Insight into the parenthood paradox: Mental health outcomes of intensive mothering. Journal of Child and Family Studies, 22(5), 614-620. doi:10.1007/s10826-012-9615-z.

Roeters, A., \& Gracia, P. (2016). Child care time, parents' well-being, and gender: Evidence from the american time use survey. Journal of Child and Family Studies, 25(8), 2469-2479. doi:10.1007/s10826-016-0416-7.

Russell, B. S., Hutchison, M., Tambling, R., Tomkunas, A. J., \& Horton, A. L. (2020). Initial challenges of caregiving during COVID-19: Caregiver burden, mental health, and the Parent-Child relationship. Child Psychiatry and Human Development, , 1-12. doi:10.1007/s10578-020-01037-x.

Shanafelt, T., Ripp, J., \& Trockel, M. (2020). Understanding and addressing sources of anxiety among health care professionals during the COVID-19 pandemic. Jama, doi:10.1001/jama.2020.5893.

Sobolewski, J., \& King, V. (2005). The importance of the coparental relationship for nonresident fathers' ties to children. Journal of Marriage and Family, 67(5), 1196-1212. doi:10.1111/j.1741-3737.2005.00210.x.

Spinelli, M., Lionetti, F., Pastore, M., \& Fasolo, M. (2020). Parents' stress and children's psychological problems in families facing the COVID-19 outbreak in italy. Frontiers in Psychology, 11 doi:10.3389/fpsyg.2020.01713. 
Statacorp. (2017). Stata statistical software: Release 15.[computer software]. College Station, TX: StataCorp LLC.

the University of Southern California. (2020). Understanding america study. Retrieved from https://uasdata.usc.edu/index.php.

U.S. Department of Labor. (2020). Unemployment insurance weekly claims. Washington, DC.

Umberson, D., Pudrovska, T., \& Reczek, C. (2010). Parenthood, childlessness, and Well-Being: A life course perspective. Journal of Marriage and Family, 72(3), 612-629. doi:10.1111/j.1741-3737.2010.00721.x. 
Table 1: Participant characteristics among UAS sample ages 25-55

\begin{tabular}{|c|c|}
\hline \multicolumn{2}{|c|}{$\begin{array}{c}\text { Total Sample }(\mathrm{N}=2524) \\
\text { Sociodemographic Characteristics }-N(\%)\end{array}$} \\
\hline \multicolumn{2}{|l|}{ Age } \\
\hline $25-34$ & $773(30.6 \%)$ \\
\hline $35-44$ & $985(39.0 \%)$ \\
\hline $45-55$ & $766(30.4 \%)$ \\
\hline \multicolumn{2}{|l|}{ Sex } \\
\hline Female & $1337(53.0 \%)$ \\
\hline Male & $1187(47.0 \%)$ \\
\hline \multicolumn{2}{|l|}{ Race } \\
\hline White & $1921(76.1 \%)$ \\
\hline Black & $320(12.7 \%)$ \\
\hline Other & $284(11.2 \%)$ \\
\hline \multicolumn{2}{|l|}{ Education Level } \\
\hline High school or less & $879(34.8 \%)$ \\
\hline $\begin{array}{l}\text { Some college or Associate's } \\
\text { degree }\end{array}$ & $650(25.8 \%)$ \\
\hline Bachelor's degree & $556(22.0 \%)$ \\
\hline Graduate degree & $439(17.4 \%)$ \\
\hline \multicolumn{2}{|l|}{ Household Income } \\
\hline Less than $30 \mathrm{k}$ & $606(24.0 \%)$ \\
\hline Less than $60 \mathrm{k}$ & $597(23.7 \%)$ \\
\hline $60 \mathrm{k}+$ & $1320(52.3 \%)$ \\
\hline \multicolumn{2}{|l|}{ Currently working } \\
\hline Yes & $1665(66.0 \%)$ \\
\hline No & $859(34.0 \%)$ \\
\hline \multicolumn{2}{|l|}{ Living with a Partner } \\
\hline Yes & $1839(72.9 \%)$ \\
\hline No & $685(27.1 \%)$ \\
\hline \multicolumn{2}{|c|}{ Household structure } \\
\hline \multicolumn{2}{|l|}{ Household Structure - N (\%) } \\
\hline Alone & $313(12.4 \%)$ \\
\hline Living with Spouse & $345(13.6 \%)$ \\
\hline Living with Kids & $125(5.5 \%)$ \\
\hline Living with Spouse and Kids & $888(35.1 \%)$ \\
\hline $\begin{array}{l}\text { Living with Significant Other } \\
\text { (SO) }\end{array}$ & $145(5.6 \%)$ \\
\hline Living with SO and Kids & $135(5.2 \%)$ \\
\hline Other & $573(22.6 \%)$ \\
\hline \multicolumn{2}{|l|}{ Number of Children - mean (se) } \\
\hline Ages $0-12$ & $0.7(0.031)$ \\
\hline Ages $12-18$ & $0.3(0.016)$ \\
\hline \multicolumn{2}{|c|}{ Mental Distress - mean (se) } \\
\hline PHQ-4 Score & $2.9(0.091)$ \\
\hline Latest CES-D score & $1.9(0.062)$ \\
\hline
\end{tabular}


Table 2: Multivariable linear regression models estimating PHQ-4 total score by 1) household structure and 2) number of children in the household, stratified by age of children. Both models are adjusted for age, sex, race, education, household income, current work status, living with a partner, and historical depressive symptoms

Outcome:

\section{Primary Predictor:}

Household structure (ref: alone)

Spouse Only

Kids Only

Spouse and Kids

Significant other only

Significant other and kids

Number of children

\# children $<-12$

\# children 13-18

Age

$35-44$

45-55

Sex (ref: female)

Male

Race (ref: white)

Black

Other

Education (ref: high school or less)

Some college/Associate's

Bachelor's

Graduate degree

Household Income (ref: <30k)

30 to less than $60 \mathrm{k}$

$60 \mathrm{k}+$

Current job (ref: Yes)

No job

Live with partner (ref: no)

Living with partner

Historical depression score (CESD-
Mental Distress

Household structure

( $\mathbf{N}=1951)$

Beta $\quad 95 \% \mathrm{CI}$

0.14

$-0.11$

0.06

0.25

0.01

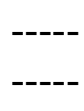

$-0.13$

$-0.43$

$-0.45$

$-1.06$

$-0.17$

$[-1.67,-0.45] *$

$[-0.76,0.43]$

$-0.03$

0.61

$[-0.55,0.48]$

$[0.09,1.13] *$

0.56

$[-0.06,1.17]$

$-0.27$

$-0.44$

$[-0.94,0.40]$

$[-1.14,0.26]$

0.55

$[0.04,1.05] *$

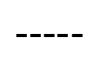

0.48

$[0.37,0.59] *$
$-0.21$

0.00

$[-0.40,-0.02]$

$[-0.27,0.26]$

Metal Distress

Number of children (by age)

( $\mathrm{N}=\mathbf{2 5 2 4})$

Beta $95 \%$ CI

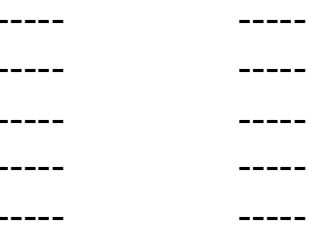

$-0.09$

$[-0.53,0.35]$

$[-0.89,-0.00] *$

$-0.45$

$[-0.67,0.04] *$

$[-1.59,-0.53] *$

$[-0.77,0.19]$

$-0.29$

$[-0.48,0.42]$

$[-0.00,0.95]$

$[-0.20,0.95]$

0.37

$[-0.65,0.50]$

$[-0.73,0.44]$

$-0.14$

$[0.24,1.12] *$

0.68

0.05

$[-0.41,0.51]$

$[0.41,0.59] *$

*denotes significance at a $\mathrm{p}<0.05$ level 


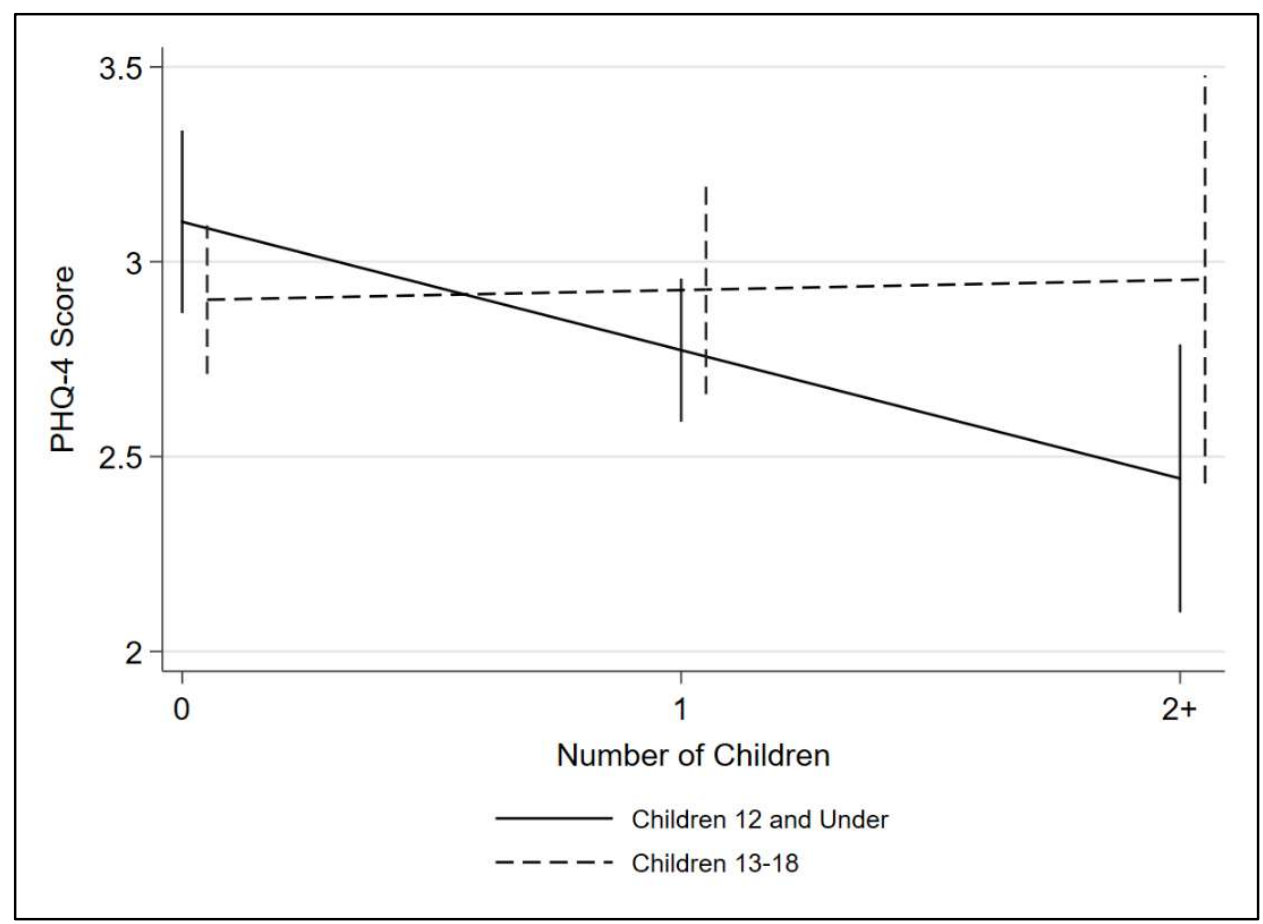

Fig. 1 Predicted PHQ-4 score and 95\% confidence interval by number of children in the household, stratified by age of children and adjusted for age, sex, race, education, household income, current work status, living with a partner, and historical depressive symptoms 
Table 3: Multivariable linear regression models with interaction terms estimating PHQ-4 total score by 1) household structure and 2) number of children in the household, stratified by age of children, and their interaction with sex. Both models are adjusted for age, race, education, household income, current work status, living with a partner, and historical depressive symptoms

Outcome:

Primary Predictor:
Mental Distress Household structure $(\mathbf{N}=1951)$

Household structure (ref: alone)

Spouse only

Kids only

Spouse and kids

Significant other only

Significant other and kids

Household structure by sex interaction

Spouse only \& Male

Kids only \& Male

Spouse and kids \& Male

Significant other only \& Male

Significant other and kids \&

Male

Number of children

\# children $<-12$

\# children 13-18

Number of children by sex

interaction

\# children $<-12$ \& Male

\# children 13-18 \& Male

Age

$35-44$

45-55

Sex (ref: female)

Male

Race (ref: white)

Black

Other

Education (ref: high school or less)

Some college/Associate's

Bachelor's

Graduate degree

Household Income (ref: $<30 \mathrm{k}$ )

30 to less than $<60 \mathrm{k}$

$60 \mathrm{k}+$

Current job (ref: Yes)

No job

\subsection{0}

$-0.11$

$-0.63$

$-1.09$

$-0.07$
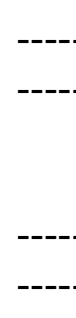

$-0.13$

$-0.41$

$[-0.64,0.38]$

$[-0.89,0.07]$

$-0.42$

$-0.12$

$[-1.13,0.90]$

$-0.14$

$[-1.62,-0.41]$

$-1.01$

$-0.15$

$[-0.74,0.43]$

$-1.04$

$-0.29$

$[-1.57,-0.51] *$

$-0.05$

0.58

$[-0.57,0.47]$

$[0.06,1.09] *$

$-0.03$

0.54

$[-0.07,1.15]$

$\begin{array}{lll}-0.3 \quad[-0.98,0.39] & -0.08\end{array}$

$-0.44$

$[-1.16,0.28]$

$-0.15$

$0.51 \quad[-0.00,1.03]$
$-0.07$

$[-0.61,0.32]$

$[-0.77,0.19]$

$[-0.48,0.42]$

$[-0.01,0.95]$

$[-0.19,0.95]$

$[-0.66,0.49]$

$[-0.73,0.43]$

0.63

$[0.18,1.08] *$

Metal Distress

$(\mathrm{N}=\mathbf{2 5 2 4})$

$[-0.39,0.13]$

$[-0.47,0.15]$

$[-0.51,0.37]$

$[-0.87,0.02]$

0.38 
Live with partner (ref: no)

Living with partner

Historical depression score

(CESD-8)

*denotes significance at a $\mathrm{p}<0.05$ level

\begin{tabular}{cccc}
---- & ---- & 0.07 & {$[-0.39,0.52]$} \\
0.47 & {$[0.36,0.59] *$} & 0.5 & {$[0.41,0.59] *$} \\
\hline
\end{tabular}

4: Multivariable linear regression models with interaction terms estimating PHQ-4 total score by 1) household structure and 2) number of children in the household, stratified by age of children, and their interaction with household income. Both models are adjusted for age, race, sex, education, current work status, living with a partner, and historical depressive symptoms

Outcome:

Primary Predictor:
Mental Distress Household structure ( $\mathbf{N}=1951)$

Beta

Significant other only

Significant other and kids

Household structure by income interaction

Spouse only \& Low income

Kids only \& Low income

Spouse and kids \& Low income

Significant other only \& Low income

Significant other and kids \& Low income

Number of children

\# children $<-12$

\# children 13-18

Number of children by income

interaction

\# children $<-12$ \& Low income

\# children 13-18 \& Low income Age

$35-44$

45-55

Sex (ref: female)

Male

Race (ref: white)

Black

Other

Education (ref: high school or less)

Some college/Associate's

$[-2.06,1.59]$
$-0.24$

$-0.22$

$-0.27$

$-0.01$

0.02

$[-1.45,0.97]$

$[-1.25,0.80]$

$[-1.16,0.62]$

$[-1.39,1.36]$

$[-1.51,1.55]$

$0.55 \quad[-0.91,2.00]$

0.15

0.44

0.41

$-0.24$

$[-1.24,1.54]$

$[-0.65,1.53]$

$[-1.22,2.05]$

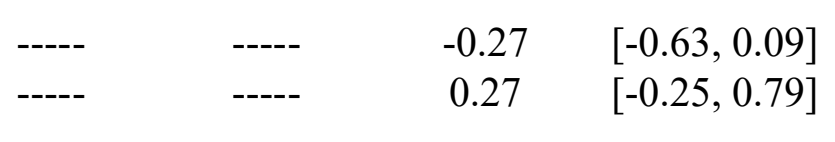

---- $\quad$-----

0.08

$[-0.32,0.48]$

$-0.41$

$[-1.01,0.18]$

$-0.13$

[-0.64, 0.37]

$-0.11$

$[-0.55,0.33]$

$-0.45$

$[-0.93,0.04]$

$-0.45$

$[-0.89,-0.00] *$

$-0.44$

$[-0.83,-0.06]$

$-0.32$

$[-0.66,0.03]$

$[-1.61,-0.38]$

$-1$

$-0.12$

*

$[-0.71,0.46]$

$-1.05$

$[-1.58,-0.52] *$

$-0.27$

$[-0.75,0.21]$

$-0.11$

$[-0.63,0.42]$

$-0.06$

$[-0.51,0.39]$ 
Bachelor's

Graduate degree

Household Income (ref: $\geq 60 \mathrm{k}$ )

Less than 60k

Current job (ref: Yes)

No job

Live with partner (ref: no)

Living with partner

Historical depression score (CESD-8) $\begin{array}{cccc}0.51 & {[0.01,1.02]^{*}} & 0.42 & {[-0.04,0.89]} \\ 0.46 & {[-0.12,1.04]} & 0.31 & {[-0.22,0.85]} \\ -0.41 & {[-1.35,0.53]} & 0.06 & {[-0.44,0.56]}\end{array}$

$0.61 \quad[0.12,1.09] * \quad 0.71 \quad[0.31,1.12] *$

---- $\quad$----- $\quad 0.04 \quad[-0.41,0.49]$

$0.49 \quad[0.38,0.60] * \quad 0.5 \quad[0.41,0.59] *$

$*$ denotes significance at a $\mathrm{p}<0.05$ level 